\title{
Nanostructure of Surface Films on Ni18P Alloy in Sulfate Solutions by the Maximum Entropy Method
}

\author{
Mariano Andrea Scorciapino, ${ }^{\dagger} \odot$ Marzia Fantauzzi, Maura Crobu, ${ }^{\ddagger}$ Gabriele Navarra, Bernhard Elsener, \\ and Antonella Rossi*৫
}

Department of Chemical and Geological Sciences, University of Cagliari, Cittadella Universitaria di Monserrato, S.P. 8 km 0.700, I-09042 Monserrato, CA, Italy

Supporting Information

ABSTRACT: NiP alloys are very often used in industry, due to their outstanding performance in corrosion and wear. Alloys with high phosphorus content $(\geq 16$ atom $\% \mathrm{P})$ are amorphous and show high corrosion resistance in both neutral and acidic solutions irrespective of the presence of chloride ions. The reason for this behavior is attributed to the formation of a "Penriched surface layer" whose exact nature is still under debate. In this work, an iterative algorithm based on the application of maximum entropy method on nondestructive angle-resolved $\mathrm{X}$-ray photoelectron spectroscopy data has been applied to the investigation of the surface layer grown on Ni18P alloys following mechanical polishing and anodic polarization in sulfate solutions. The results show that the outermost region of the examined alloy has a complex layered structure: (1) an uppermost hydrocarbon contamination layer about $1 \mathrm{~nm}$ thick, which includes also adsorbed water; (2) a nickel (poly)phosphate layer of about $1 \mathrm{~nm}$; (3) a highly phosphorus-enriched interface being about $2 \mathrm{~nm}$ thick with a marked phosphorus concentration gradient, from 70 to 20 atom \%; and (4) bulk alloy with the stoichiometric composition. These findings, together with the chemical state of the different phosphorus compounds, allow us to conclude that the high corrosion and wear resistance of $\mathrm{NiP}$ alloys might be ascribed to the presence of a thin, self-repairing nickel (poly)phosphate layer grown on a strongly P-enriched interface. Because the Auger parameter of $\mathrm{P}$ at the interface is similar to that of elemental $\mathrm{P}$, it might be also concluded that the interface is enriched in elemental phosphorus.

\section{INTRODUCTION}

$\mathrm{NiP}$ alloys have been exploited as coatings to prevent corrosion on a commercial scale since the $1950 \mathrm{~s} .{ }^{1,2}$ The interest toward nanocrystalline $\mathrm{NiP}$ alloys is due to their properties, such as hardness and good wear performance, ${ }^{3,4}$ low friction coefficient, nonmagnetic behavior, ${ }^{5}$ and high electrocatalytic activity. ${ }^{6}$ These alloys are currently used in electronic industry ${ }^{7,8}$ and $\mathrm{Ni}-\mathrm{P} / \mathrm{X}$ composites, where $\mathrm{X}=\mathrm{SiC}$, WC, $\mathrm{Al}_{2} \mathrm{O}_{3}$, and $\mathrm{TiO}_{2}{ }^{9}$ have been developed due to their improved wear and corrosion resistance. One of the most important functional properties of $\mathrm{NiP}$ alloys is their outstanding corrosion resistance in neutral and acidic solutions. In the potential range, where pure $\mathrm{Ni}$ dissolves actively, NiP alloys with near-eutectic composition of about $18-20$ atom \% $\mathrm{P}$ and an amorphous microstructure exhibit suppression of the anodic dissolution. ${ }^{10-16}$

According to the literature, this high corrosion resistance is attributed to a P-enriched surface layer, whose exact chemical nature and composition and especially the in-depth composition profile are still controversial. The improved corrosion performance was related by various authors to the presence of a nickel phosphate film, ${ }^{17,18}$ hypophosphites (called "chemical passivity"), ${ }^{19,20}$ phosphides, ${ }^{21}$ or a stable P-enriched amorphous phase. $^{21-24}$ There was agreement in stating that this layer should be localized between the surface layer and the bulk alloy. ${ }^{17-23}$
On the basis of a combined electrochemical ${ }^{10}$ and X-ray photoelectron spectroscopy (XPS) surface analytical study, ${ }^{25}$ the authors explained the corrosion behavior of NiP alloys, taking into account the "elemental" phosphorus enrichment at the interface between the surface film and the bulk alloy. However, no detailed mechanistic explanation of the formation conditions, growth kinetics, and high stability of $\mathrm{NiP}$ alloys has yet been provided, mainly because of the lack of in-depth composition profile information of the thin surface film.

Ion sputtering composition versus depth profiling yields good in-depth resolution, but this is a destructive technique and may result in artifacts, such as atom mixing, preferential sputtering of some of the specimen components, and implantation of sputtered ions. ${ }^{26,27}$ These artifacts can at times be reduced by an appropriate choice of the nature, energy, and incidence angle of impacting ions, as well as by combining ion bombardment with sample rotation. However, if sample damages have to be reduced and films of nanometer thickness have to be characterized, nondestructive methods are preferable. Among the nondestructive methods, the three-layer model approach ${ }^{18,28}$ requires a minimum of input data (the

Received: July 9, 2017

Accepted: October 31, 2017

Published: November 10, 2017 


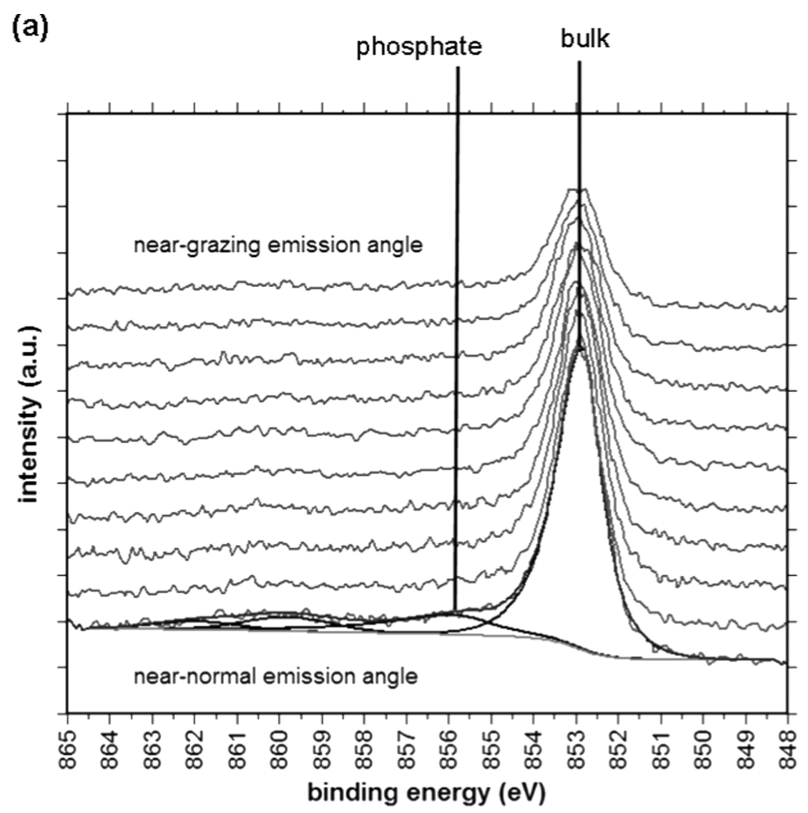

(b)

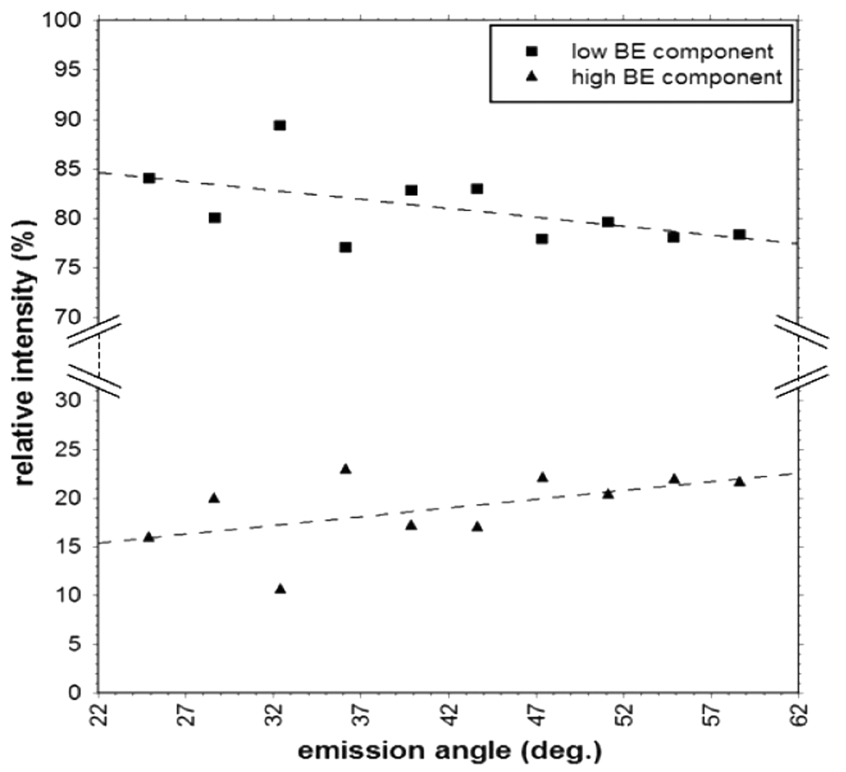

Figure 1. (a) $\mathrm{Ni} 2 \mathrm{p}_{3 / 2}$ high-resolution spectra acquired with ThetaProbe in ARXPS acquisition mode from Ni18P specimen polarized at $+0.1 \mathrm{~V}$ SCE in $0.1 \mathrm{M} \mathrm{Na}_{2} \mathrm{SO}_{4}$ for $1 \mathrm{~h}$ (with curve fitting) and (b) relative intensity vs emission angle calculated from the ARXPS data.

acquisition of XPS image at only one angle), but the information on film composition is averaged over the film thickness, thus assuming the presence of an in-depth homogeneous film.

The in-depth distribution of the elements present in surface films might be also obtained exploiting the method developed by Tougaard. ${ }^{29}$ This method requires the acquisition of reference spectra recorded on pure reference compounds, but it is limited to a maximum of three reference spectra that can be used to simulate the sample spectrum. ${ }^{28}$ The small numbers of structures that can be verified and of the structural parameters obtainable (six) represent the limit of this method when it is applied to more complex systems. ${ }^{30}$

Angle-resolved X-ray photoelectron spectroscopy (ARXPS) is considered a very convenient method for determining indepth composition profiles of thin films, ${ }^{31}$ as it is nondestructive, a particular advantage if chemical-state information is important. ${ }^{32}$ However, obtaining the concentration versus depth information from the intensity versus angle XPS data requires the inversion of a Laplace transform ${ }^{31}$ that leads to more than one solution ${ }^{32}$ (ill-posed problem). Furthermore, the noise of ARXPS data strongly affects the results. ${ }^{31,32}$ The reconstruction is based on the assumption of model profiles but, due to the presence of noise, many models matching experimental data might exist. ${ }^{32,33}$ For this reason, it is not possible to reconstruct the profiles by simply minimizing the weighted sum-of-square differences between calculated and measured data, especially if the surface layer is complex and consists of many components. However, the reconstruction of the depth profiles from ARXPS data is achievable by the maximum entropy method (MEM). ${ }^{26,33-38}$

The importance of MEM reconstruction starting by ARXPS data, for example, in semiconductors field, was highlighted in the last years by Yonamoto $^{39}$ who applied MEM data processing not only to ARXPS data, but also to thermally stimulated current, isochronal annealing (IA), and thicknessdependent CV (cyclic voltammetry) data, proving that MEM allows an easy extraction of important information for understanding the physics of devices.

The impact of the noise in angle-resolved data ${ }^{31,32}$ can be controlled by implementing a suitable algorithm. ${ }^{40}$ The first MEM algorithm was developed by the research group and applied to the nondestructive in-depth composition profile of oxy-hydroxide layers on iron surfaces. ${ }^{38}$ Then, an MEM algorithm was developed and combined with the previous version in a unique iterative protocol. ${ }^{40}$ A large number of numerical experiments were carried out showing that the iterative protocol is suitable for reconstruction of many different depth profile topologies with up to eight components. ${ }^{40}$ In this work, it has been applied to a real case, the characterization of the P-enriched surface films grown on a Ni18P alloy. The results of the in-depth composition profile enabled the proposal of a new model for the mechanism of surface film formation and the rationalization of the high corrosion and wear resistance of the NiP alloys.

The main advantage of MEM reconstruction starting from ARXPS data is that it is possible to obtain the composition versus depth distribution of all species optimizing the IMFP of the emitted electrons; in our knowledge, there are no other examples that are based on the optimization of the IMFP.

\section{RESULTS}

Electrochemical Results. The electrochemical behavior of the NiP alloy in near-neutral solutions was previously studied, ${ }^{10}$ and the results are here briefly summarized. The high corrosion resistance of $\mathrm{NiP}$ alloys is confirmed by anodic polarization curves and by the corrosion current densities ranging between 0.5 and $0.7 \mu \mathrm{A} / \mathrm{cm}^{2}$; the inhibition of oxygen reduction is inferred by dynamic cathodic polarization curves in deaerated solutions. The potentiostatic polarization curves at $0.1 \mathrm{~V}$ saturated calomel electrode (SCE) in deaerated $0.1 \mathrm{M}$ sodium sulfate solution showed that the current density decreased upon time according to a square root law. This finding suggests a diffusion-controlled reaction. Full results are provided in ref 10 . In the present study, further investigations have been carried 


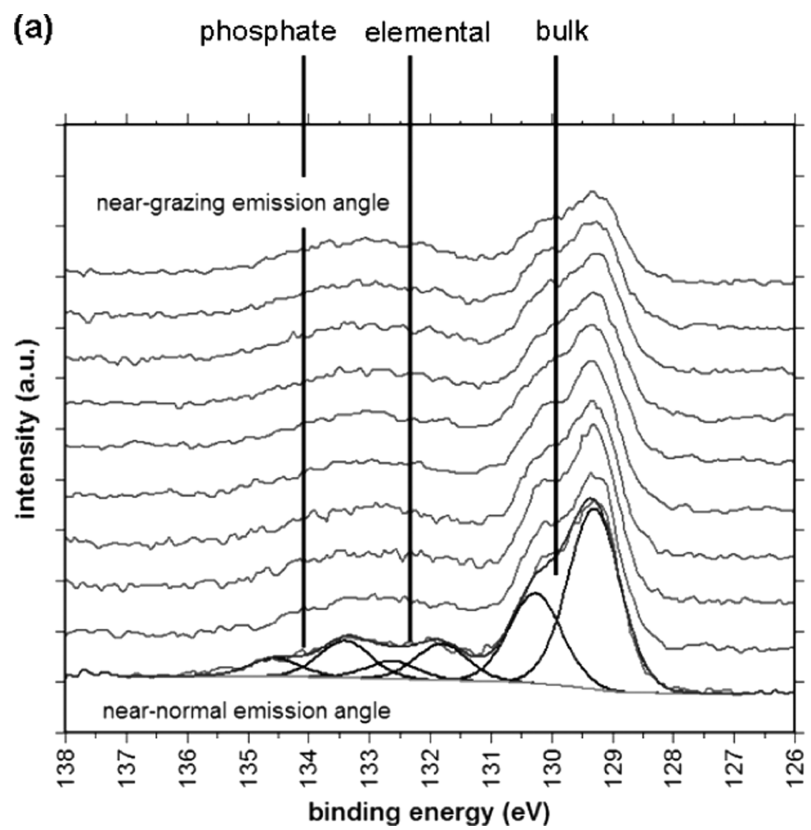

(b)

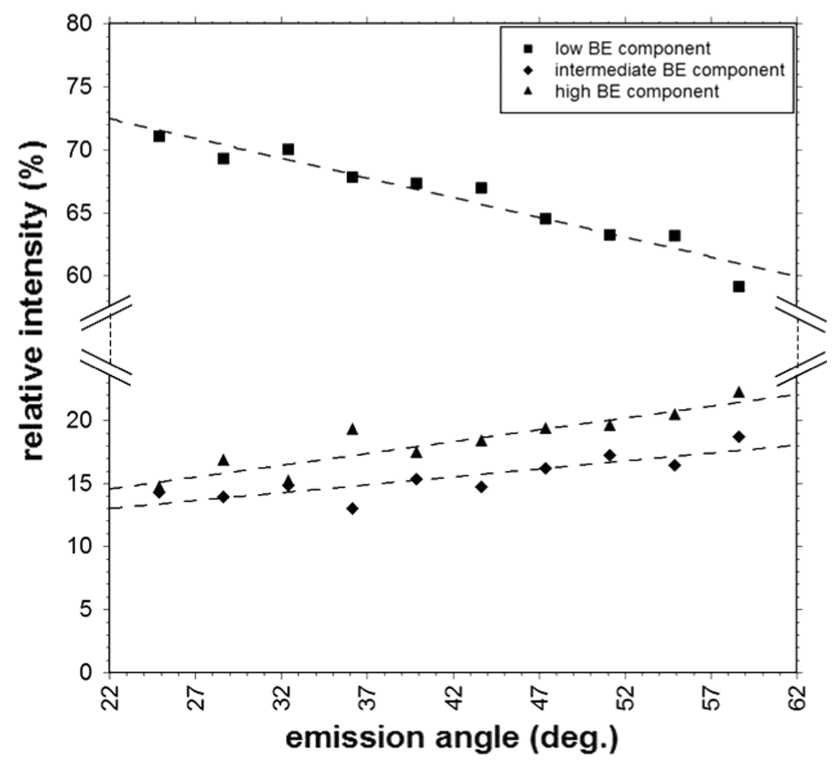

Figure 2. (a) P 2p high-resolution spectra acquired with ThetaProbe in the ARXPS acquisition mode from Ni18P specimen polarized at $+0.1 \mathrm{~V}$ SCE in $0.1 \mathrm{M} \mathrm{Na}_{2} \mathrm{SO}_{4}$ for $1 \mathrm{~h}$ (with curve fitting) and (b) relative intensity vs emission angle calculated from the ARXPS data.
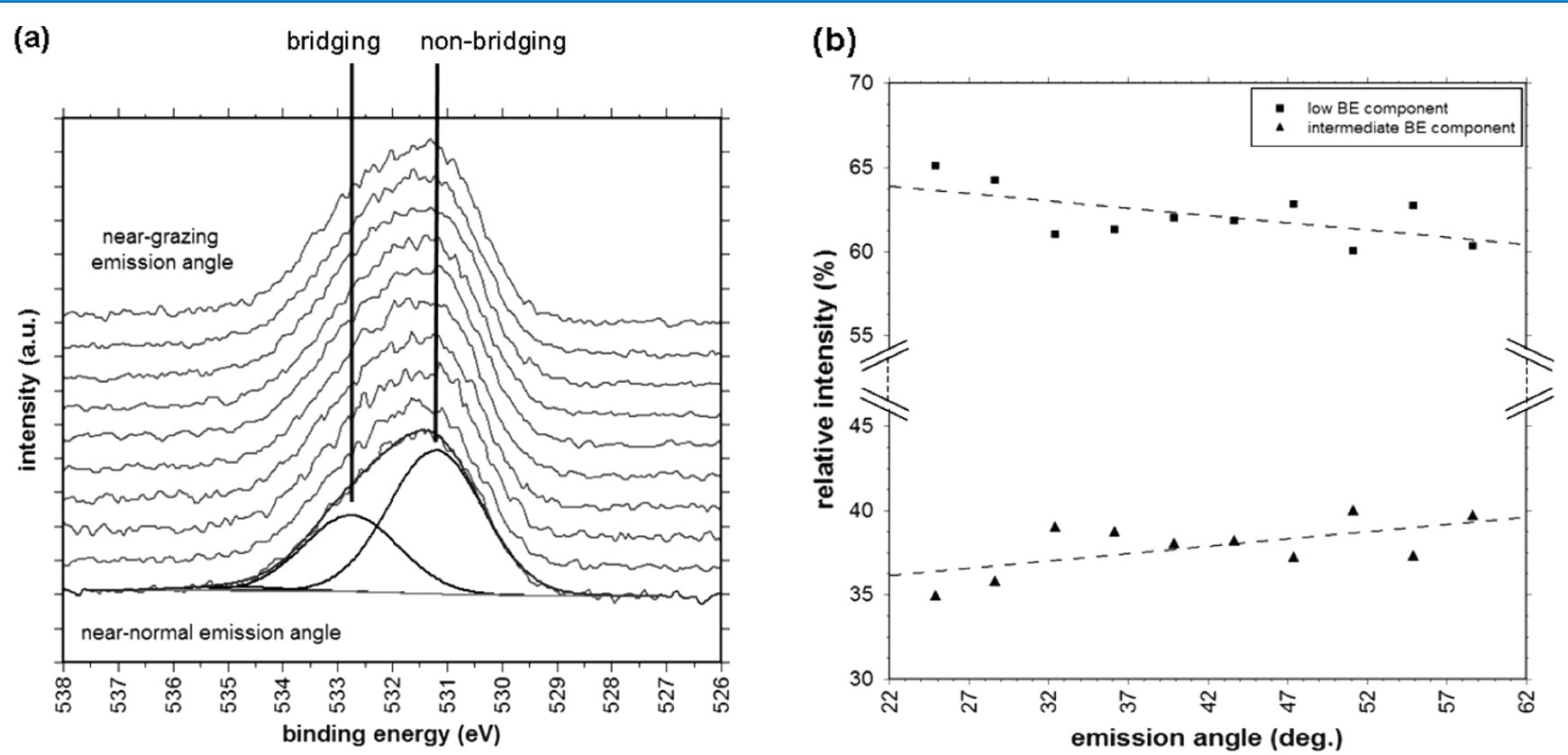

Figure 3. (a) $\mathrm{O} 1 \mathrm{~s}$ high-resolution spectra acquired with ThetaProbe in the ARXPS acquisition mode on a Ni18P specimen polarized at $+0.1 \mathrm{~V}$ SCE in $0.1 \mathrm{M} \mathrm{Na}_{2} \mathrm{SO}_{4}$ for $1 \mathrm{~h}$ (with curve fitting) and (b) relative intensities vs emission angle calculated from the ARXPS data.

out for detailing the in-depth composition profiles of the Ni18P alloy exposed to solutions using ARXPS and applying an MEM protocol presented in ref 40. This allows focusing on the structural chemistry at the surface film-bulk alloy interface.

ARXPS Results. Figures $1-4$ show the examples of the high-resolution spectra of $\mathrm{Ni} 2 \mathrm{p}_{3 / 2}, \mathrm{P} 2 \mathrm{p}, \mathrm{O} 1 \mathrm{~s}$, and $\mathrm{C} 1 \mathrm{~s}$ acquired in angle-resolved mode on a Ni-P sample polarized at $+0.1 \mathrm{~V} \mathrm{SCE}$ in a $0.1 \mathrm{M} \mathrm{Na}_{2} \mathrm{SO}_{4}$ solution for $1 \mathrm{~h}$. The changes in signal intensities and shape as a function of the emission angle are plotted for 10 emission angles together with an example of fitted spectrum. The curve fitting of the $\mathrm{Ni} 2 \mathrm{p}_{3 / 2}$ signal (Figure 1a) was performed using two components. ${ }^{8,18,25}$ The most intense peak, found at $853.1 \mathrm{eV}$ with its satellite at $860.4 \mathrm{eV}$, was assigned, in agreement with the literature ${ }^{18}$ and on the basis of the results published in a previous work of the authors, ${ }^{8,25}$ to nickel in the bulk alloy, whereas the second peak at $857.0 \mathrm{eV}$ and its satellite at $863.2 \mathrm{eV}$ were attributed to $\mathrm{Ni}^{2+}$ in nickel phosphate or polyphosphates. $8,18,25,41,42$ The same parameters were adopted for fitting the high-resolution spectra acquired in the standard mode (Figure S2) for validating the procedure. The relative intensity of the two signals versus the emission angle (Figure 1b) shows some scatter due to the fact that the spectra were acquired using the ARXPS acquisition lens mode. However, a trend to a smaller value of the component "nickel bulk" and an increase of the component "nickel phosphate" can be observed at higher emission angle. 
Similar trends were also confirmed by the spectra acquired following the mechanical polishing protocol and after 3 and 14 $\mathrm{h}$ of potentiostatic polarization in sulfate solution.

$P 2 p$ Region. The angle-resolved high-resolution spectra of the $\mathrm{P} 2 \mathrm{p}$ signal (Figure 2a) were processed using three components, i.e., three doublets with the $\mathrm{P} 2 \mathrm{p}_{3 / 2}$ at $129.5(0.1)$ $\mathrm{eV}, 132.0(0.1) \mathrm{eV}$, and 133.7 (0.1) eV (Figure 2a bottom) in agreement with literature results. ${ }^{18,20,25}$ Each doublet assigned to $\mathrm{P} 2 \mathrm{p}_{3 / 2}$ and $\mathrm{P} 2 \mathrm{p}_{1 / 2}$ was fitted constraining the area ratio to $2: 1^{27}$ and constraining the binding energy difference to $0.81 \mathrm{eV}$. On the basis of the literature, ${ }^{18,20}$ other works of the authors, ${ }^{27}$ and high-resolution spectra acquired in the standard mode (Figure S3), the doublet at $129.5 \mathrm{eV}$ was assigned to phosphorus in the bulk alloy. The doublet at $133.7(0.1) \mathrm{eV}$, assigned to $\mathrm{P}$ in phosphate, was shifted to about $0.3-0.4 \mathrm{eV}$ toward high binding energy values compared to the value measured on mechanically polished NiP sample $(133.3 \mathrm{eV})$. A similar binding energy shift was detected between the reference pyrophosphate (133.6 (0.1) eV) and the orthophosphate $(133.3 \mathrm{eV}){ }^{8}$

As far as the intermediate $\mathrm{BE}$ component is concerned, its chemical state has been under discussion since a long time. The presence of $\mathrm{P}(\mathrm{I})$ and $\mathrm{P}(\mathrm{III})$ in unidentified compounds and the possible formation of hypophosphite were assumed ${ }^{19,20}$ at the interface between the surface film and the bulk alloy. These assignments were based only on the chemical shift of the photoelectron spectrum. In this work, the modified Auger parameter $\alpha$ calculated as $\alpha=\mathrm{BE}(2 \mathrm{p})+\mathrm{KE}(\mathrm{KLL})^{43-45}$ and the Wagner plot are used to identify the chemical state of phosphorus. This approach allowed the authors, on the basis of the results presented in a previous paper, ${ }^{25}$ to assign the doublet at $132.0 \mathrm{eV}$ to phosphorus with a chemical environment very close to that of elemental phosphorus and to confirm the attribution of the lower and higher BE component.

The relative intensity of the peak assigned to $\mathrm{P}$ in the bulk alloy $(129.5 \mathrm{eV})$ decreases at high emission angle (Figure $2 \mathrm{~b}$ ), whereas the relative intensities of the other two phosphorus components at 132.0 and $133.7 \mathrm{eV}$ were found to increase (Figure 2b).

The same tendency was exhibited by the spectra obtained on polarized samples for 3 and $14 \mathrm{~h}$ as well as after the mechanically polished sample.

$O$ 1s Region. Figure 3a presents the angle-resolved highresolution spectra of $\mathrm{O}$ 1s together with the example of a fitted spectrum. Two components were detected at binding energies of 531.5 and $532.9 \mathrm{eV}$. The former was attributed to nonbridging oxygen and the latter to bridging oxygen in polyphosphates. At this $\mathrm{BE}$ value, there might also be a small contribution from adsorbed water. The presence of a contribution of organic contamination at $531.5 \mathrm{eV}$ due to the contact with the laboratory atmosphere cannot be ruled out even if, after both mechanical polishing and electrochemical tests, the samples were transferred in the vacuum chamber in less than $3 \mathrm{~min}$.

The $\mathrm{O}$ 1s spectra obtained on NiP alloys following mechanically polishing (Figure S4) only two components at $531.5(0.1) \mathrm{eV}$ and $533.4(0.1) \mathrm{eV}$ were revealed. ${ }^{8}$ The spectra acquired after 1 and $3 \mathrm{~h}$ of potentiostatic polarization did not exhibit differences in the relative intensity (Figure S5); on the contrary, polarization for $14 \mathrm{~h}$ resulted in an increase of the most intense peak at about $531 \mathrm{eV}$, whereas the peak at 532.9 eV decreased.
In the MEM calculations, only the total O 1 s intensity was considered to not influence depth profile reconstruction on the basis of $\mathrm{O} 1 \mathrm{~s}$ curve fitting and assignments. This is justified by the relative intensities of the two components that do not vary, within experimental error, with emission angle (Figure $3 \mathrm{~b}$ ), and by the fact that the MEM protocol ${ }^{40}$ has proved to be able to properly work on an element present in two adjacent layers with different concentrations. ${ }^{40}$

C 1s Region. C 1s spectra (Figure 4) are found to be the convolution of two signals: one at $284.8(0.1) \mathrm{eV}$ and the other

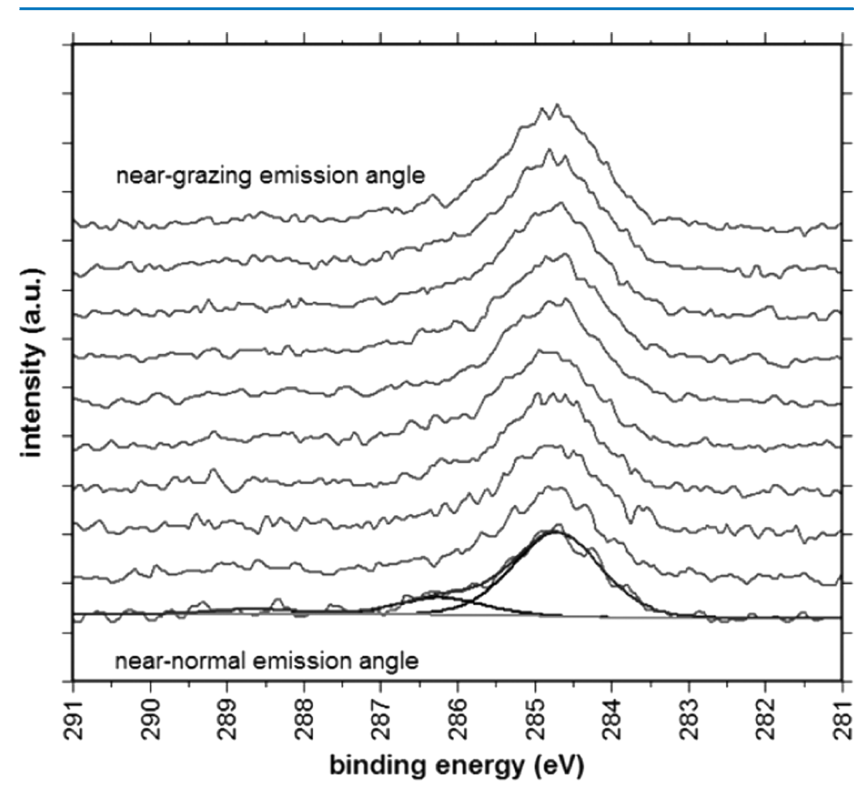

Figure 4. $\mathrm{C}$ 1s high-resolution spectra acquired with ThetaProbe in ARXPS acquisition mode from Ni18P specimen polarized at $+0.1 \mathrm{~V}$ SCE in $0.1 \mathrm{M} \mathrm{Na}_{2} \mathrm{SO}_{4}$ for $1 \mathrm{~h}$.

at $286.6(0.1) \mathrm{eV}$. Their binding energy values as well as the relative intensities did not vary with the polarization potential and time. The peak at $284.8 \mathrm{eV}$ originates from aliphatic carbon of the adventitious contamination. The peak at $286.6 \mathrm{eV}$ might be assigned to a carbon atom bonded to an oxygen atom. Figure S6 shows the high-resolution spectra obtained in the standard lens mode.

The relative intensities of the two components remained constant with the emission angle, and similarly to what was done for the oxygen, it was decided to use the intensity of the whole spectral region in MEM protocol application.

Application of the MEM Protocol to Real NiP Samples. The MEM protocol was presented in detail in a previous paper, ${ }^{40}$ where it was emphasized that its novelty is related to the possibility of calculating the thicknesses of the layers and their composition versus depth optimizing the IMFP of the emitted electrons. It was applied to mechanically polished Ni18P alloys and the same alloys polished and polarized for 1, 3 , and $14 \mathrm{~h}$ at $+0.1 \mathrm{~V} \mathrm{SCE}$ in neutral sulfate solution. Here, the specific case of the Ni18P alloy, polarized for $1 \mathrm{~h}$, is taken as an example. The details of the data processing are provided in the Experimental/Data Processing section. Figure 5a shows the experimental apparent concentration diagram (ACD) calculated for emission angles from 25 to $57^{\circ}$ on the basis of the ARXPS data (Figures 1-4).

Literature on NiP alloys with phosphorus content higher than 16 atom \% agrees on the statement that the observed high 


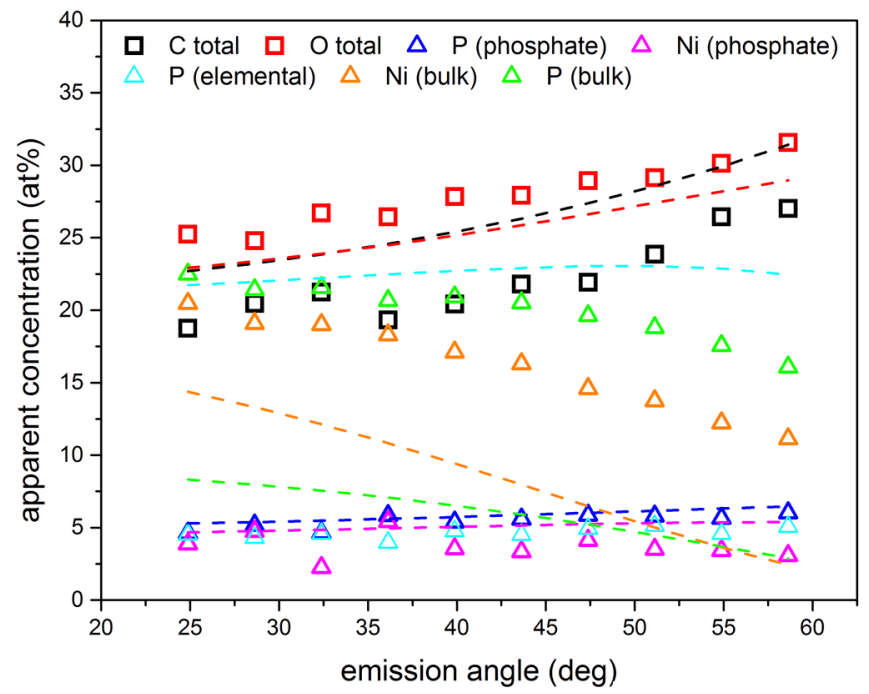

Starting hypothesized profile:

1) contamination layer

Thickness $1 \mathrm{~nm}$

Composition: 80 at\% C, 20 at\% O

2) phosphate layer

Thickness $1 \mathrm{~nm}$

Composition: 62 at\% O, 23 at\% Ni, 15 at\% P

3) Interface

Thickness $1 \mathrm{~nm}$

Composition: 100 at\% P

4) bulk - alloy

Composition: 82 at\% Ni, 18 at\% P

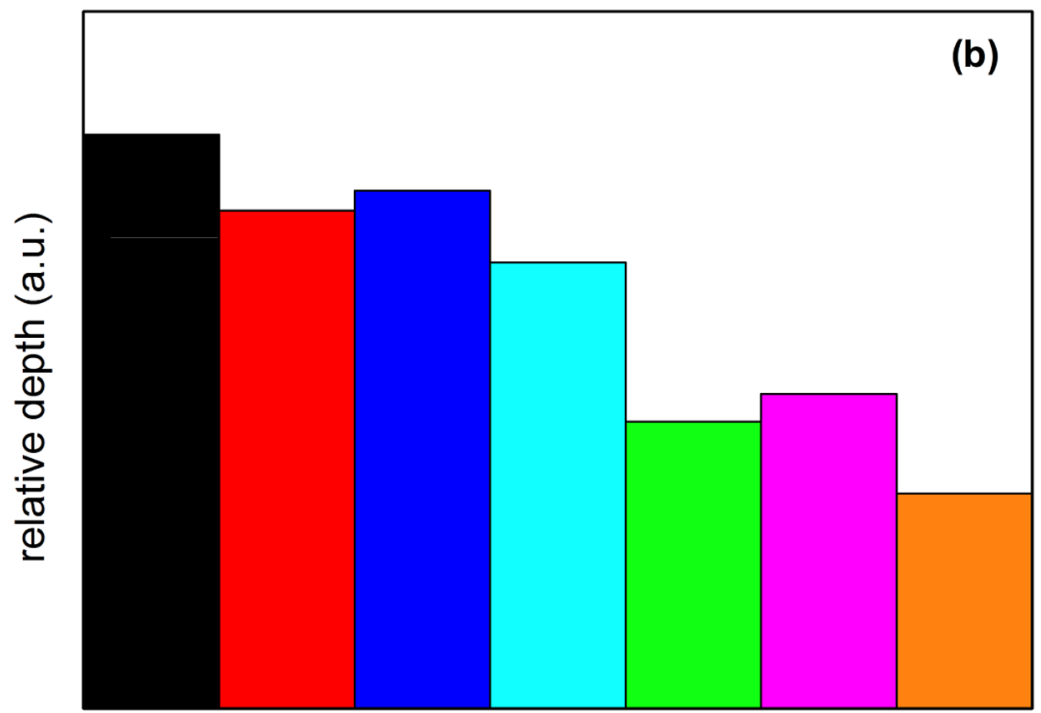

C total $\mathrm{O}$ total $\mathrm{P}$ phos $\mathrm{P}$ elem $\mathrm{P}$ bulk Ni phos Ni bulk

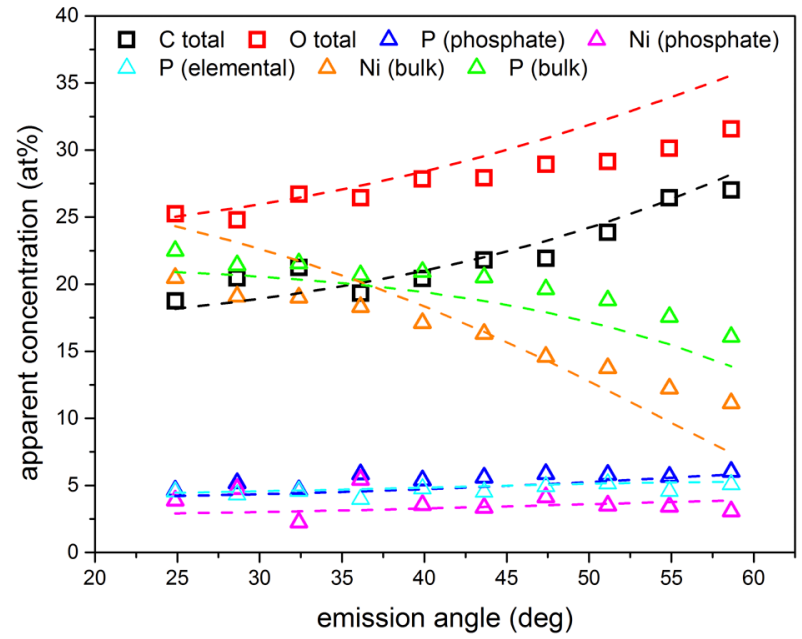

Refined profile:

1) contamination layer

Thickness $0.8 \mathrm{~nm}$

Composition: 80 at\% C, 20 at\% O

2) phosphate layer

Thickness $1 \mathrm{~nm}$

Composition: 73 at\% O, 18 at\% Ni, 9 at\% P

3) Interface

Thickness $1 \mathrm{~nm}$

Composition: 45 at\% P - bulk, 35 at\% Ni -

bulk, 20 at\% Pelementl

4) bulk - alloy

Composition: 82 at\% Ni, 18 at\% P

(a) 
corrosion resistance can be attributed to a "phosphorusenriched surface film" that strongly limits alloy dissolution and not to an oxide-type passivation. ${ }^{10,25}$ Despite different assignments of the phosphorus species in the literature, ${ }^{25}$ a layered structure of a phosphorus species (hypophosphite, ${ }^{20}$ orthophosphate, $^{18}$ phosphide, $^{21}$ or phosphate ${ }^{25}$ ) on top of a Penriched interface can be proposed here as the starting model. Evidences derived from Auger electron spectroscopy depth profiling $^{19}$ and glow discharge optical microscopy ${ }^{46}$ also support the presence of this P-enriched interface.

The relative depth plot RDP (Figure 5b) shows the highest values for carbon and oxygen: this suggests that a contamination layer is present at the outermost surface. Looking at different phosphorus compounds, the sequence Pphosphate, P-elemental, and P-bulk can be clearly observed. The same can be revealed for nickel, where Ni-phosphate is external with respect to the Ni-bulk.

Application of Tougaard's method to the same Ni-P samples ${ }^{8,25}$ resulted in an outer phosphate layer having a thickness of $1 \mathrm{~nm}$ and a $1 \mathrm{~nm}$ thick intermediate P-enriched layer present at the interface with the bulk alloy. The content of phosphorus at the interface resulted to be about 55 atom \%.

The starting hypothesis for the depth profile was a layered structure with an outermost contamination layer (thickness, 1 $\mathrm{nm}$ ), a stoichiometric nickel phosphate layer of $1 \mathrm{~nm}$ thickness, and a $1 \mathrm{~nm}$ thick P-enriched interface on a bulk alloy with nominal composition (Table 1). On the basis of this initial assumption, the ACD plot was calculated and is shown by the dotted lines in Figure 5a. It appears obvious that this hypothesis was not conforming to the actual depth profile (root-meansquare difference (RMSD) was 9.1 atom \%). For instance, the

Table 1. Quantitative Information on the Composition Depth Profile of a Ni18P Alloy (Polarized for $1 \mathrm{~h}$ in $+0.1 \mathrm{~V}$ SCE in $\left.\mathrm{Na}_{2} \mathrm{SO}_{4} 0.1 \mathrm{M}\right)^{a}$

\begin{tabular}{|c|c|c|c|}
\hline & $\begin{array}{c}\text { starting } \\
\text { hypothesis }\end{array}$ & $\begin{array}{l}\text { simulator } \\
\text { refinement }\end{array}$ & $\begin{array}{c}\text { MEM } \\
\text { algorithm }\end{array}$ \\
\hline \multicolumn{4}{|l|}{ contamination layer } \\
\hline $\begin{array}{l}\text { thickness } \\
(\mathrm{nm})\end{array}$ & 1.0 & 0.8 & $1.0 \pm 0.6$ \\
\hline $\mathrm{C}$ (atom \%) & 80 & 80 & $78 \pm 12$ \\
\hline $\mathrm{O}($ atom \%) & 20 & 20 & $22 \pm 12$ \\
\hline \multicolumn{4}{|l|}{ phosphate layer } \\
\hline $\begin{array}{l}\text { thickness } \\
(\mathrm{nm})\end{array}$ & 1.0 & 1.0 & $1.2 \pm 0.6$ \\
\hline $\mathrm{Ni}$ (atom \%) & 23 & 18 & $11 \pm 3$ \\
\hline $\mathrm{P}($ atom \%) & 15 & 9 & $23 \pm 9$ \\
\hline $\mathrm{O}($ atom \%) & 62 & 73 & $66 \pm 12$ \\
\hline \multicolumn{4}{|l|}{ P-enriched interface } \\
\hline $\begin{array}{l}\text { thickness } \\
(\mathrm{nm})\end{array}$ & 1.0 & 1.0 & $2.0 \pm 0.6$ \\
\hline $\mathrm{Ni}$ (atom \%) & 0 & 35 & $54 \pm 6$ \\
\hline $\begin{array}{l}\mathrm{P}_{\text {el. }} \\
\text { (atom \%) }\end{array}$ & 100 & 20 & $13 \pm 3$ \\
\hline $\begin{array}{l}\mathrm{P}_{\text {bulk }} \\
\text { (atom \%) }\end{array}$ & 0 & 45 & $33 \pm 3$ \\
\hline \multicolumn{4}{|l|}{ bulk } \\
\hline $\begin{array}{l}\text { thickness } \\
(\mathrm{nm})\end{array}$ & \multicolumn{2}{|l|}{ semi-infinite } & \\
\hline composition & Ni18P & & \\
\hline
\end{tabular}

${ }^{a}$ The different phases of the MEM protocol: starting hypothesis, best layered structure after refinement in the simulator routine, and after application of the MEM algorithm. thickness of the P-enriched interface appeared to be too large because all calculated apparent concentrations for P-elemental were higher than the experimental values. The bulk $\mathrm{Ni}$ and $\mathrm{P}$ concentrations appeared too small at all of the emission angles. Thus, the starting hypothesis had to be modified. As the ACD data are normalized to $100 \%$ at each of the emission angles, during this refinement, every modification would affect all of the other concentrations. An iterative procedure (simulator routine $\mathrm{H}^{40}$ ) was applied to find the best concentration-depth profile, as described in Experimental/Data Processing section.

The best layered structure was found to be a contamination layer of $0.8 \mathrm{~nm}$ thickness, a nickel phosphate layer of $1 \mathrm{~nm}$ thickness, and a phosphorus-enriched interface of $1 \mathrm{~nm}$ thickness on the bulk alloy (Table 1). The ACD of this refined structure is shown in Figure $5 c$ together with the experimental data. Compared to Figure 5a, a clearer improvement can be observed (RMSD decreased to 1.6 atom \%).

As input for the application of the MEM algorithm to the experimental ACD data (Figure 5a,c), only the layer thicknesses of the best layered depth profile (Table 1) were used, whereas the chemical composition of individual layers was not taken into account, that is, each layer started with equal amount of all of the species present. The only constrain was the known bulk composition of the alloy (Ni18P). MEM reconstructed depth profile of the same alloy polarized for $1 \mathrm{~h}$ at $+0.1 \mathrm{~V}$ SCE is shown in Figure 6a, confirming the layered structure with the sequence: contamination layer $(1.0 \mathrm{~nm})$, nickel phosphate layer $(1.2 \mathrm{~nm})$ on top of the strongly phosphorus-enriched interface $(2.0 \mathrm{~nm})$, and the bulk. Compositions of the different layers are provided in Table 1. The correspondent apparent concentration diagram (Figure $6 \mathrm{~b}$ ) is in fairly good agreement with apparent concentration versus emission angle ARXPS data, and the RMSD has the lowest value of 1.2 atom \%.

The anomalous "peak" present in the P-bulk curve at the phosphate/bulk interface (Figure 6a) is not "real" but a systematic artifact of the MEM algorithm: this particular feature appeared every time when the algorithm tried to reproduce an interface enriched in the lower-concentration component of the bulk, even in the numerical experiments. ${ }^{8,40}$

Nevertheless, thickness and composition of the enriched layer were always reproduced within the expected accuracy 8,40 as the relative concentration of a certain species in a given layer is obtained from the area (and not from the height) of the corresponding curve in the depth profile.

Figure 7 shows a schematic diagram of 1 h-polarized Ni18P alloy depth profile reconstructed by the MEM protocol.

Mechanically Polished Samples. Table 2 shows quantitative results of the MEM reconstructed depth profile for mechanically polished samples. The sequence contamination layer $(1.0 \mathrm{~nm})$, nickel phosphate layer $(0.9 \mathrm{~nm})$, and phosphorus-enriched interface $(1.0 \mathrm{~nm})$ was found. Nickel phosphate layer on the mechanically polished samples shows with good accuracy the stoichiometric composition of $\mathrm{Ni}_{3}\left(\mathrm{PO}_{4}\right)_{2}$ standard $(23$ atom $\% \mathrm{Ni}, 15$ atom \% P, 62 atom $\% \mathrm{O}$ ) formed as reaction product during mechanical polishing. The thickness of P-enriched interface was found to be about 1 $\mathrm{nm}$, with composition of 40 atom $\%$ phosphorus.

Polarized Samples. The quantitative results of MEM reconstructed depth profile following the potentiostatic polarization for 1,3 , and $14 \mathrm{~h}$ are reported in Table 2. The thicknesses of all of the three layers (contamination, phosphate and P-enriched layer) do not significantly vary with polarization time. The composition of the nickel phosphate layer is enriched 

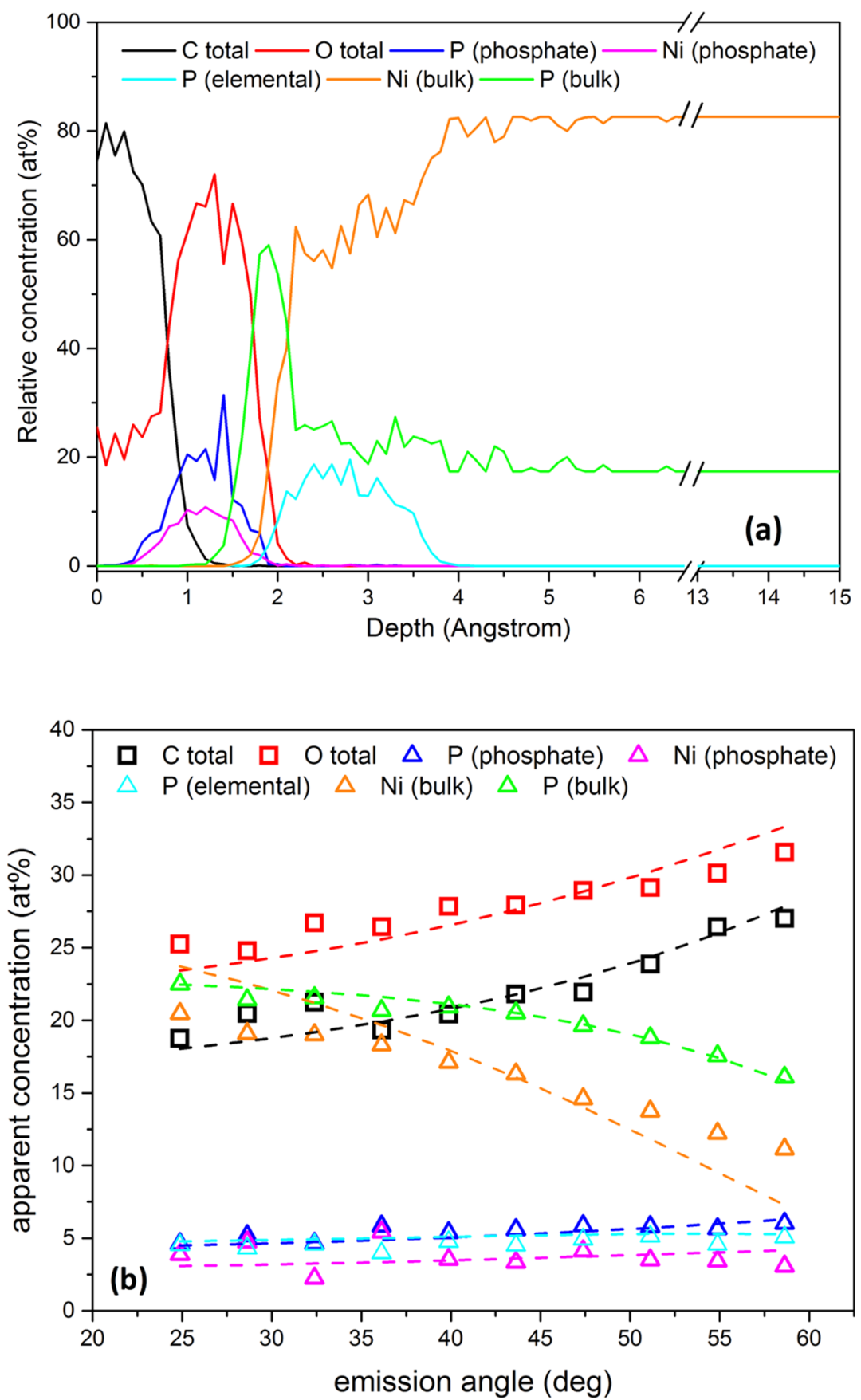

Figure 6. (a) MEM reconstructed depth profile of a Ni18P alloy after $1 \mathrm{~h}$ polarization at $+0.1 \mathrm{~V}$ SCE in $0.1 \mathrm{M} \mathrm{Na}_{2} \mathrm{SO}_{4}$ and (b) apparent concentration diagram from experimental data (symbols) and recalculated MEM data (dashed lines).

with phosphorus and depleted in nickel compared to the expected stoichiometry of $\mathrm{Ni}_{3}\left(\mathrm{PO}_{4}\right)_{2}$. For P-enriched interface, a thickness of ca. $2 \mathrm{~nm}$ and a composition of nearly 50 atom \% of $\mathrm{P}$ was found; thus, exposure to an electrolyte and electrochemical polarization leads to a strong alloy dissolution and associated $\mathrm{P}$ enrichment at the interface.

Additionally, the MEM reconstruction revealed that phosphorus at the P-enriched interface between the surface layer and the bulk alloy of all samples is not present in a unique chemical state, but present in the bulk alloy with chemical bonds to nickel and at the interface as elemental phosphorus, meaning that phosphorus appears here to be mainly surrounded by other phosphorus atoms.

\section{DISCUSSION}

The present work reports for the first time full quantitative information on the composition of the layers in function of depth of NiP alloys. These findings constitute the base for providing a rational model of the mechanism of formation and of the nature of the P-enriched surface layer and might also contribute to shed a light on the high corrosion resistance of this peculiar alloy system.

Uncertainties on Concentration and Depth Resolution. According to Cumpson, ${ }^{31}$ ARXPS inversion is sensitive primarily to the precision rather than accuracy of peak intensity measurement. This means that the accuracy of the final depth profile will depend on the accuracy of elemental sensitivity 


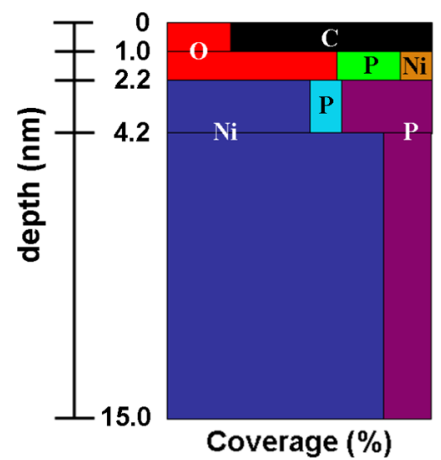

Figure 7. Schematic diagram of depth profile of a 1 h-polarized Ni18P alloy, reconstructed with MEM protocol.

factors, primary beam variability, and others, no more than in ordinary "single emission angle" XPS systematic uncertainties.

On the other hand, signal-to-noise ratio was found to intrinsically affect the relative depth resolution $(\Delta z / z)$ of an MEM reconstructed depth profile. ${ }^{31}$ Experimental ARXPS data presented in this study have an $\mathrm{S} / \mathrm{N}$ ratio belonging to the range indicated by Cumpson ${ }^{31}$ as that achievable in "careful peak intensity measurements," i.e., region of practical significance for depth profiling.

The maximum expected uncertainty was found to be 20 and $30 \%$ for thickness and concentration, respectively, for tests performed on self-assembled monolayer on gold with known composition depth profile (unpublished data). This result was also confirmed by large series of numerical experiments, where a random error was introduced in the data to take into account the influence of experimental error on MEM protocol accuracy. ${ }^{40}$

Finally, another possible source of uncertainty, especially on overlayer thickness determination, is the roughness of sample surface. In this work, this effect was neglected following investigations by Gunter et al. ${ }^{47}$ and Oswald. ${ }^{48}$

According to Gunter and co-workers, ${ }^{47}$ an error lower than $10 \%$ affects the estimation of thickness of overlayers grown on rough substrates when the roughness is not taken into consideration. Oswald ${ }^{48}$ evaluated the effect of surface roughness by computer simulations, taking into account the presence of islands on smooth overlayers. He concluded that, if the structure size was smaller than the attenuation length of the photoelectrons, the amount of the overlayer material or its thickness could be calculated, with a systematic underestimation of the quantification in the range up to $5 \%$.

Concentration-Depth Profile of Ni18P Samples. The ACD diagram corresponding to the starting hypothesis of the depth profile (Figure 5a) showed an RMSD from experimental data of 9.1 atom \%. After the application of the simulator routine (Figure $5 \mathrm{c}$ ), the RMSD of the calculated ACD data decreased to 1.6 atom \%, indicating the efficiency of the routine itself to produce a refined depth profile. When concentrations were removed from the latter, i.e., the composition of all of the four layers was assumed to be homogeneous and only layers thicknesses were used to build up the initial profile, an increase of the RMSD up to 5.4 atom \% was observed. This is solely due to a deviation from real layers composition because their thickness did not change. On the other hand, being this last RMSD value only $60 \%$ of that corresponding to the starting hypothesis, this result suggests that layer thicknesses were varied properly and thus we obtained really improved starting conditions. Finally, after MEM reconstruction, both layer thickness and composition were further refined up to an RMSD equal to 1.2 atom \%, i.e., lower than that corresponding to the simulator profile.

Results of the concentration-depth profile obtained from ARXPS data using the MEM protocol ${ }^{40}$ (Figures 1-4) show that after anodic polarization at $+0.1 \mathrm{~V} \mathrm{SCE}$ in $0.1 \mathrm{M} \mathrm{Na}_{2} \mathrm{SO}_{4}$, as well as after mechanical polishing, a nickel phosphate overlayer (thickness, ca. $1 \mathrm{~nm}$ ) forms. A new result is that MEM depth profile reconstruction also enabled to calculate the stoichiometry of this phosphate layer (Table 2), thus providing important new insight into the surface film on $\mathrm{NiP}$ alloys (see section below).

The MEM composition depth profile (Figure 6a) also reveals the formation of a P-enriched layer at the interface between the phosphate layer and the bulk alloy, as schematically displayed in Figure 7. The presence of the P-enriched layer was reported or suggested by several other groups, ${ }^{1-22}$ mostly without specifying its thickness and composition. The MEM protocol

Table 2. Depth Profile Parameters Obtained by Applying the MEM Protocol to a Series of Ni18P Alloy Samples after 1, 3, and $14 \mathrm{~h}$ of Polarization at $+0.1 \mathrm{~V} \mathrm{SCE}$ in $0.1 \mathrm{M} \mathrm{Na}_{2} \mathrm{SO}_{4}{ }^{a}$

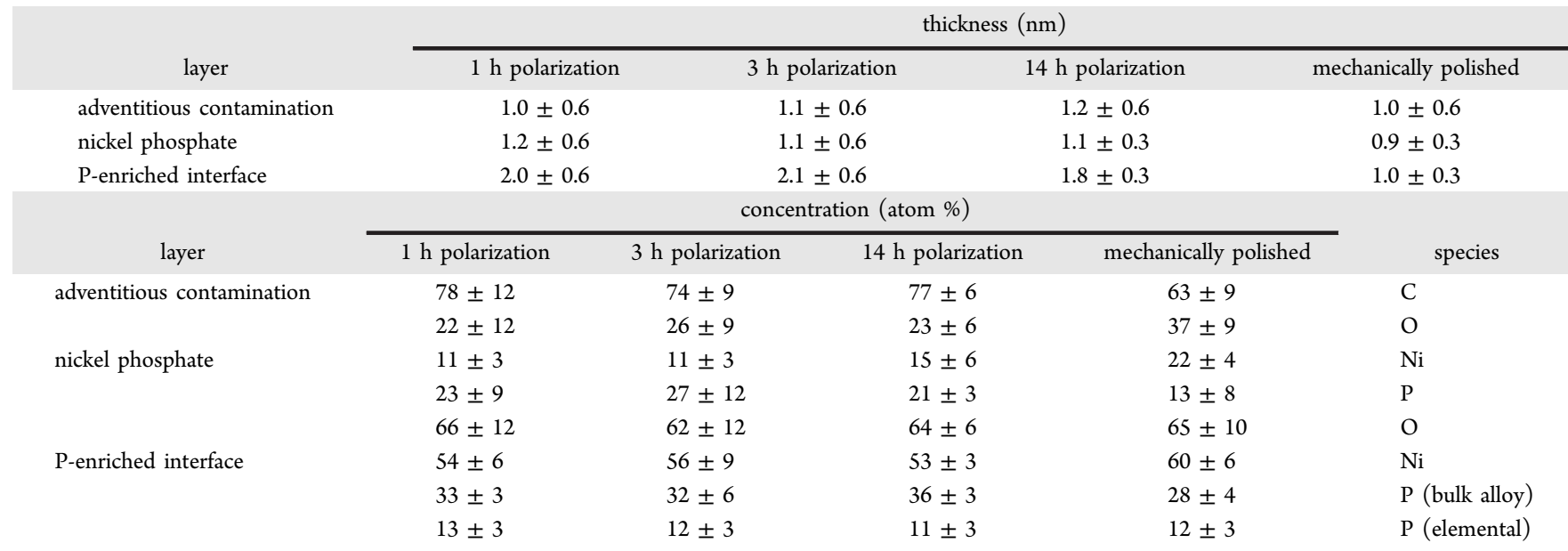

${ }^{a}$ Data obtained on mechanically polished sample are also listed. Results are the mean and standard deviation calculated over three independent measurements. 
applied in this work has allowed quantifying both its thickness and composition. The thickness of this P-enriched interface was estimated to be about $2 \mathrm{~nm}$ for polarized samples and about 1 $\mathrm{nm}$ for the mechanically polished ones (Table 2). Composition depth profile obtained from MEM reconstruction (Figure 6a) made it possible to distinguish the chemical state of phosphorus $8,23,25$ in the P-enriched interface as phosphorus in the bulk NiP alloy (with chemical bonds to nickel) and phosphorus in the elemental chemical state (chemical bonds only to other P atoms). For a total concentration of 46 atom \% at the P-enriched interface, about 34 atom \% might be considered as $\mathrm{P}$ surrounded by $\mathrm{Ni}$ atoms and the rest, about 12 atom \%, could be assigned to elemental P (Table 2).

Finally, the concentration-depth profile from MEM reconstruction allowed taking into account also the adventitious contamination layer. The contamination layer was calculated to be $1 \mathrm{~nm}$ thick and its composition resulted to be 80 atom \% C and 20 atom $\% \mathrm{O}$.

The composition as a function of depth was revealed to remain unchanged with the potentiostatic polarization time, within the accuracy of the MEM protocol. ${ }^{40}$

Chemical Nature of the Phosphate Layer. The thin phosphate layer detected on the surface of the alloy by the ARXPS data (nickel Ni 2p, Figure 1a; phosphorus P 2p, Figure 2a) was revealed on both mechanically polished and polarized Ni18P sample surfaces. The concentration-depth profile reconstructed with the MEM protocol ${ }^{40}$ shows this layer to have a thickness of ca. $1 \mathrm{~nm}$ (Table 2), in good agreement with results from Tougaard's method, which yielded 0.9 (0.1) nm. ${ }^{8,25}$ In addition to thickness, the composition of the phosphate layer was calculated using the MEM algorithm (Table 2). On mechanically polished samples, an inorganic nickel phosphate layer with practically nominal composition of $\mathrm{Ni}_{3}\left(\mathrm{PO}_{4}\right)_{2}$ was found (stoichiometric composition: $\mathrm{Ni}, 23$ atom \%; P, 15 atom \%; O 62 atom \%). On the other hand, the Ni/P/ $\mathrm{O}$ concentration ratio of 1:2:5 for the polarized Ni18P samples suggests a composition close to nickel polyphosphate (Table 2).

This result was also suggested by XPS images of $\mathrm{O}$ 1s region (Figure 3a), where the presence of "nonbridging" ( $\mathrm{BE}=531.1$ $\mathrm{eV})$ and "bridging" oxygen $(\mathrm{BE}=532.2 \mathrm{eV})$ could be distinguished, as the chain structure of polyphosphates ${ }^{43}$ presumes. However, because total $\mathrm{O} 1 \mathrm{~s}$ intensity was used in the MEM protocol, the nonbridging/bridging oxygen ratio was not resolved in the reconstructed depth profile. However, the curve fitting of O 1s ARXPS images acquired on the polarized samples was also performed using a model function to take into account the contribution due to adsorbed water and constraining the position of all of the other components; in this way, it was possible to determine a nonbridging/bridging oxygen intensity ratio equal to about 1.8 . This value was found in long-chain phosphate. ${ }^{42}$ On the contrary, $\mathrm{O} 1 \mathrm{~s}$ spectra recorded on the mechanically polished sample ${ }^{8}$ (not shown) did not present any bridging oxygen contribution and the nonbridging $\mathrm{O}$ to $\mathrm{P}$ concentration ratio of 5.0 could be considered compatible with the reference $\mathrm{Ni}_{3}\left(\mathrm{PO}_{4}\right)_{2}$ compound within the MEM accuracy. ${ }^{8,40}$

This difference between mechanically polished and polarized phosphate layer is further confirmed by the P $2 \mathrm{p}$ chemical shift. The $\triangle \mathrm{BE}$ between the phosphate component recorded on polarized and nonpolarized samples was found to be the same as that observed between the reference nickel pyrophosphate and orthophosphate (see above).
Summarizing, on mechanically polished samples, a thin layer of nickel orthophosphate, about $1 \mathrm{~nm}$ thick, is present. On the polarized samples, thus in the region of current arrest and high corrosion resistance, the presence of the previously suggested $^{17,18}$ nickel orthophosphate could be ruled out. On these samples, a long-chain polyphosphate layer is present. These long-chain polyphosphates, indeed, show very low solubility in water and exhibit excellent tribological properties. 49,50

Influence of Polarization Time. The parameters investigated in this work for elucidating the effect of time were the binding energy values, the intensity ratio of the various phosphorus $\mathrm{P} 2 \mathrm{p}$ species, the thickness of both the polyphosphate layer and the P-enriched interface, and the composition of the polyphosphate layer. The first two were found to be independent of the polarization time within the accuracy of MEM (Table 2), whereas small changes upon longer polarization have been revealed in the composition of the polyphosphate film (Table 2).

A further indication of changes in the polyphosphate layer is found in the $\mathrm{O}$ 1s spectra collected at near-normal emission angles. The nonbridging/bridging oxygen ratio was found to increase from 1.8 after $1 \mathrm{~h}$ of polarization to about 3.0 following $14 \mathrm{~h}$ of polarization at $+0.1 \mathrm{~V} \mathrm{SCE}$ in $0.1 \mathrm{M} \mathrm{Na}_{2} \mathrm{SO}_{4}$ solution. This change is mainly due to an increase in the intensity of the nonbridging oxygen component. The nonbridging oxygen to Pphosphate intensity ratio at $14 \mathrm{~h}$ of polarization is higher $(+40 \%)$ than that after 1 or $3 \mathrm{~h}$. These results may be ascribed to changes in the polyphosphate chain length. ${ }^{41}$

An increased phosphorus concentration resulting from the initial preferential dissolution of nickel might contribute, at longer times, to a more diffusion-limited dissolution of nickel through the P-enriched interface, as observed with electrochemical experiments during potentiostatic polarization or corrosion at the open-circuit potential in $0.1 \mathrm{M} \mathrm{NaCl} .^{10}$ The proof for this hypothesis is lacking, as surface analytical data of samples immersed for long times at the free corrosion potential are not (yet) available.

The experiments performed at $+0.1 \mathrm{~V}$ SCE, thus at the higher end of the current arrest in polarization curves, ${ }^{10,25}$ showed a slow current increase after $1-3 \mathrm{~h}$ of polarization ${ }^{10,25}$ associated with the appearance of localized black spots after 14 $\mathrm{h}$ in both chloride and sulfate solutions. ${ }^{10}$ The same features were observed after anodic potentiodynamic scans to $0.6 \mathrm{~V}$ SCE and have also been reported in earlier works. ${ }^{15,21,51}$ These features cannot be considered as "normal" pitting corrosion because the black spots were found to occur also in sulfate solutions when chloride ions were absent. ${ }^{10}$ The black spots seem to be randomly distributed and not related to the morphological structure of the underlying alloy. One possible explanation for these features might be the local breakdown of the polyphosphate film as indicated by the changing nonbridging/bridging oxygen ratio and by the $\mathrm{O}$-nonbridging to $\mathrm{P}$ phosphate ratio at longer polarization times. This local breakdown does not seem to occur at lower potentials ${ }^{25}$ (e.g., $-0.1 \mathrm{~V} \mathrm{SCE}$ ).

Model of Formation and Dissolution of Protective Surface Film on Ni-P Alloys. The proposed mechanism of reaction of the $\mathrm{NiP}$ alloy when immersed in a sulfate solution at $\mathrm{pH} 6.3$ is the following: both nickel and phosphorus tend to dissolve in the electrolyte solution ${ }^{50}$ as $\mathrm{Ni}^{2+}$ and $\mathrm{H}_{2} \mathrm{PO}_{3}{ }^{-}$. Due to the fact that nickel dissolves faster than phosphorus, ${ }^{10,20,22}$ a progressive enrichment of $\mathrm{P}$ results at the alloy surface. The 
dissolution of nickel in this early stage $(<1 \mathrm{~h}$ at $+0.1 \mathrm{~V} \mathrm{SCE})$ can be, based on electrochemical results, described as a diffusion-limited process. ${ }^{10,25}$ The nickel dissolution causes the thickening of the P-enriched layer and also compositional changes. As a result, phosphorus is present in two different chemical states, one corresponding to phosphorus still bonded to $\mathrm{Ni}$ atoms and the other resulting in $\mathrm{P}$ atoms surrounded by other phosphorus atoms that it might be described as elemental P. According to this model substantiated by the surface analytical results, the hypothesis of formation of an orthophosphate, hypophosphite, or phosphide layer can be ruled out in agreement with the previous findings. ${ }^{25}$

Dihydrogenated phosphite anions eventually formed might be further oxidized ${ }^{52}$ and form dihydrogenated phosphate anions $\left(\mathrm{H}_{2} \mathrm{PO}_{4}^{-}\right)$that might react to form polyphosphate chains that are characterized by low water solubility. The thin polyphosphate layer formed on top of the P-enriched layer might also act as a barrier between the alloy surface and the electrolyte solution so that the corrosion reaction is slowed down.

The appearance of the "black spots" that become visible upon prolonged polarization time might be interpreted in terms of breakdown of the polyphosphate layer. The surface film seems to be in dynamic transformation: the long-chain phosphates might be converted into soluble dihydrogenated orthophosphate and thus readily dissolved. Thus, several "channels" might be generated on the surface film resulting in a local destruction of the protective layer. The oxygen might then diffuse through them to reach the surface underneath, causing the dissolution of nickel into the solution. The formation of these channels is in agreement with the findings of other authors who explained in this way the breakdown of the protective surface film of $\mathrm{NiP}$ alloys. ${ }^{15}$ The evidence provided by the MEM composition versus depth profile is that the average composition and thickness of the surface layer remains unchanged upon time, supporting that any further dissolution of the alloy is counterbalanced by a self-healing process that results in the repair of the surface films.

\section{CONCLUSIONS}

The results of the maximum entropy method (MEM) approach applied to ARXPS data obtained on Ni18P alloys after mechanical polishing and anodic polarization in sulfate solution indicate the presence of a complex layered structure. Four layers were identified by this approach with different thicknesses and compositions: an outermost contamination layer made of organic carbon and oxygen; a second thin layer (about $1 \mathrm{~nm}$ thick) made of nickel (poly)phosphate, and a highly phosphorus-enriched interface zone of about $2 \mathrm{~nm}$ thick with a marked concentration gradient. Finally, the bulk of the alloy is revealed with the nominal composition. From this work, it can be concluded:

Quantitative in-depth composition profiles in the nanometer range are provided by the MEM protocol taking into account for the first time the variation of IMFP values of the photoelectrons within the layers. The protocol works successfully, reducing the root-mean-square difference (RSMD) between experimental data and model profile from 9.1\% for the initial guess to $1.2 \%$ after MEM application.

The MEM depth profile substantiates the diffusioncontrolled dissolution mechanism of the $\mathrm{NiP}$ alloys, providing evidence of the presence of phosphorus not coordinated to nickel at the interface.
The long-chain nickel polyphosphate layer detected at the surface is acknowledged to have a low solubility in water. This layer might act as a barrier at the surface of the alloy and hinders the dissolution of nickel ions. Its presence explains the excellent tribological properties of NiP alloys, and the complex structure characterized by the polyphosphate film on top of a layer containing $\mathrm{P}-\mathrm{P}$ bonds provides an experiment-supported explanation of the outstanding corrosion resistance of Ni18P alloys.

Further work is needed to ascertain if the proposed model rationalizing the high corrosion resistance of the $\mathrm{NiP}$ alloys holds at open-circuit (immersion) conditions.

\section{EXPERIMENTAL/DATA PROCESSING}

NiP Alloys Preparation and Characterization. NiP coatings were prepared by electroless deposition using a commercial nickel hypophosphite bath operating at $\mathrm{pH} 4.8$ and $88{ }^{\circ} \mathrm{C}$ (Galvanic, Wädenswil, $\mathrm{CH}$ ). The nickel plating bath was composed of nickel sulfate $\left(\mathrm{NiSO}_{4} \cdot 6 \mathrm{H}_{2} \mathrm{O}\right)$ and sodium hypophosphite $\left(\mathrm{NaH}_{2} \mathrm{PO}_{2} \cdot 2 \mathrm{H}_{2} \mathrm{O}\right)$ together with complexing agents and stabilizers. The plating rate was $10-12 \mu \mathrm{m} / \mathrm{h}$, and the coating thickness was $15-20 \mu \mathrm{m}$. Further details on the deposition process are given in refs $8,10,25$. The coatings had phosphorus content of 18.4-19.0 atom \% and X-ray amorphous structure. The results of morphological characterization are reported elsewhere. ${ }^{25}$

Sample Preparation. Electrochemical measurements were performed using an EG\&G 273 potentiostat/galvanostat controlled by M352 software and a traditional three-electrode cell; a saturated calomel electrode was used as reference (SCE, potential $+0.242 \mathrm{~V}$ vs $\mathrm{NHE}$ ). More information on the setup are given elsewhere. ${ }^{8,10,25}$

Potentiostatic polarizations were performed for 1,3 , and $14 \mathrm{~h}$ at $+0.1 \mathrm{~V} \mathrm{SCE}$ in deaerated $0.1 \mathrm{M} \mathrm{Na}_{2} \mathrm{SO}_{4}$ solution, on specimens kept at the open-circuit potential (OCP) for $15 \mathrm{~min}$. More details are given in refs $10,25$.

Mechanically polished Ni18P samples were analyzed by XPS, and the same samples were also analyzed after electrochemical tests. Details on mechanical polishing conditions are given in ref 25.

XPS Analysis and Data Processing. XPS characterization of NiP alloys was carried out using a ThetaProbe spectrometer (Thermo Fisher Scientific Inc., East Grinstead, U.K.). The analysis chamber is under ultra-high-vacuum conditions, and the pressure during spectra acquisition was always lower than 5 $\times 10^{-6} \mathrm{~Pa}$.

A monochromatic $\mathrm{Al} \mathrm{K} \alpha$ source $(1486.6 \mathrm{eV})$ was used at 4.7 $\mathrm{mA}$ and $15 \mathrm{kV}(70 \mathrm{~W})$ that resulted in a $300 \mu \mathrm{m}$ spot size. Both high-resolution and survey spectra were acquired in fixed analyzed transmission mode, and the pass energy was set to 100 $\mathrm{eV}$. Calibration of the binding energy scale was accomplished using reference materials SCAA90 of $\mathrm{Cu}, \mathrm{Ag}$, and Au following an in situ argon ion sputtering necessary to remove the contamination and the thin oxide layer. ${ }^{53}$ The full width at halfmaximum (FWHM) of the $\mathrm{Ag} 3 \mathrm{~d}_{5 / 2}$ line, under these experimental conditions, was found to be $0.83 \mathrm{eV}$. ThetaProbe intensity/energy response function (IERF) was determined following instructions reported in the spectrometer user guide, using a polynomial fit to a $\log _{10}\left(\mathrm{Ag} 3 \mathrm{~d}_{5 / 2}\right.$ peak area/PE) versus $\log _{10}(\mathrm{RR})$ plot, where $\mathrm{RR}$ is the retard ratio defined as the ratio between the electron kinetic energy and the pass energy (KE/ $\mathrm{PE})$. 
The experiments were carried out in standard mode at $53^{\circ}$ emission angle and in the so-called angle-resolved mode. This mode allows the collection at 16 different emission angles, ranging from 24.88 to $81.13^{\circ}$. Spectra obtained at emission angles above $60^{\circ}$ were not used in this work because no correction of elastic scattering was applied ${ }^{54,55}$ (see section IMFP Calculation). The ThetaProbe spectrometer allows the parallel collection of angle-resolved data by means of a $2 \mathrm{D}$ detector. Compared to serial ARXPS, which requires the tilting of the sample and the acquisition of the spectra at each angle, the data acquisition is faster; furthermore, the sample is not moved during the parallel acquisition, thus ensuring that the position of the analyzed point is not changing during the analysis. For insulating samples, also the charge compensation conditions in parallel ARXPS experiments are maintained constant. $^{56}$

The sputtering conditions were $3 \mathrm{kV}$ and $1 \mu \mathrm{A}$. Neutralization of the samples was carried out using the neutralizer supplied by Thermo Fisher by flooding the sample with low-energy electrons and low-energy ions.

CASA XPS software (Casa Software Ltd., U.K.) was exploited for data processing. The curve fitting model using Gaussian-Lorentzian product functions followed ShirleySherwood background subtraction. ${ }^{8}$ Peak areas, which in the following are indicated with the term intensities, were corrected for Scofield's photoionization cross section, ${ }^{57}$ angular asymmetry function, ${ }^{58}$ and ThetaProbe intensity/energy response function (IERF). The angular asymmetry function was calculated using a routine that accounts for the various emission $\gamma$ angles in the angle-resolved acquisition mode. The corrected intensities were normalized to 1 for each emission angle. These normalized intensities were plotted versus emission angle to construct the apparent concentration diagram (ACD).

The maximum entropy method (MEM) implemented by the authors and reported in detail in a previous paper ${ }^{40}$ was then applied for nondestructive depth profile reconstruction.

Starting Depth Profile. The MEM protocol proposed by the authors requires an initial depth profile as input, which should be typically based on (i) the apparent concentration diagrams (ACD), (ii) relative depth plot (RDP), and (iii) results obtained with Tougaard's method. ${ }^{29}$

$A C D$ Diagrams. The experimental ACD is calculated as described above and represents the basic input for a preliminary hypothesis of the depth profile. A simulator routine has been implemented in the MEM protocol ${ }^{40}$ to calculate the atomic concentration diagram corresponding to the hypothesis of the depth profile. The simulator is applied to look for the "best layered depth profile" (i.e., a simple wafer model without any concentration gradients between adjacent layers) whose calculated ACD best reproduced the experimental one. This actually is an iterative procedure to minimize the difference (root-mean-square difference, RMSD) between experimental ACD and the calculated ACD for the theoretical depth profile. The number of numerical layers $(0.1 \mathrm{~nm}$ each $)$ in the simulator routine was taken as 10 times the maximum IMFP of the bulk alloy components, considering the electrons to travel only through the alloy itself (i.e., P 2p $1.9 \mathrm{~nm}$, thus 190 layers). During simulator processing, whenever the depth profile was modified, a new set of IMFP values (one for each component) was calculated accordingly as described below. At the end of the simulator routine, the number of numerical layers was decreased to 150 , with $\mathrm{ACD}$ data difference with respect to their initial values $<1 \%$.

Relative Depth Plot. Due to the noise in the experimental data, the relative position of a chemical species in the depth profile may be difficult to be obtained from ACD diagrams. The relative depth plot (RDP), a histogram calculated as the ratio of the relative concentration at near grazing to that at near-normal emission angle for each of the species, can be helpful in achieving a rough initial estimate of the depth profile: the lower the relative depth, the deeper the species. Because differences in effective attenuation length of the various species have a nonnegligible effect on the resulting relative depths, RDP should be used only as qualitative support to other information on the sample derived from the literature and/or other experimental evidence. However, by keeping constant both the material being crossed by photoelectrons and the emission angle, effective attenuation length mainly depends on the kinetic energy of the photoelectrons. Thus, while relative depth of different elements may lead to misinterpretation, RDP data obtained for different components of the same XPS signal provide a correct indication of relative depth of the different chemical species of the same element.

Tougaard's Method. This method ${ }^{29}$ is totally independent of MEM application because it starts from standard-mode survey spectra instead of involving high-resolution angleresolved data. In this work, it was found to be a very useful tool to perform a first depth profile reconstruction of $\mathrm{NiP}$ alloys. $^{8,25}$

Finally, taking into account literature information about polarized NiP alloys, RDP data, and results from Tougaard's method, ${ }^{8,25}$ the "best layered structure" was obtained with the simulator routine by comparison with experimental ACD data, and IMFP values were determined accordingly. ${ }^{40}$ This profile was then used as starting input for the MEM algorithm, taking only the thickness but discarding the composition of the layers. ${ }^{40}$

IMFP Calculation. The G1 equation ${ }^{59}$ implemented in the NIST "Standard Reference Database 71 " software ${ }^{60}$ was used to calculate the electron mean free paths (IMFP). In Figure S1, the IMFP values versus kinetic energy are plotted, taking into account four different situations:

(1) the electron is traveling through an homogeneous adventitious organic layer (density $\left.=1 \mathrm{~g} / \mathrm{cm}^{3}\right)$ composed of $\mathrm{O}(\alpha$ atom $\%)$ and $\mathrm{C}(\beta$ atom $\%)$;

(2) the electron is traveling in $\mathrm{Ni}_{3}\left(\mathrm{PO}_{4}\right)_{2}$ (experimentally measured density, $1.6 \mathrm{~g} / \mathrm{cm}^{3}$ );

(3) the electron is traveling in pure red $\mathrm{P}^{59}$

(4) the electron is traveling in the Ni18P alloy (density, 7.75 $\left.\mathrm{g} \mathrm{cm}^{-3}\right)$.

For all of the chemical species detected, four IMFP values were calculated because photoelectrons traveled through each of the materials listed above; the calculated IMFPs were used as starting values for the simulator routine ${ }^{40}$ which provides the actual IMFP as the average of the IMFPs in all of the four mentioned materials, using thickness and composition of each layer in the profile as weighting factors.

As mentioned, spectra acquired at emission angles larger than $60^{\circ}$ were not used in the MEM reconstruction because increasing effect of elastic scattering causes deviation from the Beer-Lambert law that is at the basis of MEM formalism. ${ }^{40}$

Actually, the effective attenuation length (EAL) should be used. It is usually $10-40 \%$ smaller than IMFP due, again, to 
elastic scattering events. Unfortunately, the derived EALs generally have large uncertainties because of inadequate knowledge of film morphology and thickness. ${ }^{61}$ As a result, EAL values deduced from universal curves or experimental studies should be regarded only as rough guides. ${ }^{61}$ Moreover, it was shown that calculations for quantitative analysis require knowledge of IMFP rather than EAL and that the use of the latter may result in considerable errors. ${ }^{62}$

\section{ASSOCIATED CONTENT}

\section{S Supporting Information}

The Supporting Information is available free of charge on the ACS Publications website at DOI: 10.1021/acsomega.7b00958.

IMFP values of electrons; high-resolution XPS images of $\mathrm{Ni} 2 \mathrm{p}_{3 / 2}, \mathrm{P} 2 \mathrm{p}, \mathrm{O} 1 \mathrm{~s}$, and $\mathrm{C} 1 \mathrm{~s}$ of mechanically polished samples; high-resolution $\mathrm{O}$ 1s XPS images acquired after polarization at $+0.1 \mathrm{~V} \mathrm{SCE}$ in $0.1 \mathrm{M} \mathrm{Na}_{2} \mathrm{SO}_{4}$ solution for 1,3 , and $16 \mathrm{~h}$ (PDF)

\section{AUTHOR INFORMATION}

\section{Corresponding Author}

*E-mail: rossi@unica.it. Phone: +39-070-675-4464. Fax: +39070-675-4456.

\section{ORCID}

Mariano Andrea Scorciapino: 0000-0001-7502-7265

Antonella Rossi: 0000-0002-5151-2634

\section{Present Addresses}

${ }^{\ddagger}$ Mettler-Toledo GmbH, Process Analytics, Im Hackacker 15, CH-8902 Urdorf, Switzerland (M.C.).

${ }^{\dagger}$ Department of Biomedical Sciences, Biochemistry Unit, University of Cagliari, Cittadella Universitaria di Monserrato, S.P. $8 \mathrm{~km} \mathrm{0.700,} \mathrm{I-09042} \mathrm{Monserrato,} \mathrm{CA,} \mathrm{Italy} \mathrm{(M.A.S.).}$

\section{Notes}

The authors declare no competing financial interest.

\section{ACKNOWLEDGMENTS}

This work was undertaken with financial support from the University of Cagliari and the Italian Ministry of the Education and University (MIUR). The authors acknowledge the company Galvanic AG, Wädenswil (Switzerland), for the production of the electroless deposited NiP alloys.

\section{REFERENCES}

(1) Brenner, A.; Couch, E. G.; Williams, E. K. Electrodeposition of alloys of phosphorus with nickel or cobalt. J. Res. Natl. Bur. Stand. 1950, 44, 109-122.

(2) De Minjer, C. H.; Brenner, A. Studies on electroless nickel plating. Plating 1957, 44, 1297-1305.

(3) Jeong, D. H.; Erb, U.; Aust, K. T.; Palumbo, G. The relationship between hardness and abrasive wear resistance of electrodeposited nanocrystalline Ni-P coatings. Scr. Mater. 2003, 48, 1067-1072.

(4) Palaniappa, M.; Seshadri, S. K. Friction and wear behavior of electroless $\mathrm{Ni}-\mathrm{P}$ and $\mathrm{Ni}-\mathrm{W}-\mathrm{P}$ alloy coatings. Wear 2008, 265, 735740.

(5) Press, M. R.; Khanna, S. N.; Jena, P. Electronic structure, magnetic behavior, and stability of Ni-P. Phys. Rev. B 1987, 36, 5446.

(6) Zhang, H.; Gu, C.; Huang, M.; Wang, Z.; Tu, J. Anchoring threedimensional network structured $\mathrm{Ni}-\mathrm{P}$ nanowires on reduced graphene oxide and their enhanced electrocatalytic activity towards methanol oxidation. Electrochem. Commun. 2013, 35, 108-111.

(7) Grimsley, S. Applications for electroless nickel - A 'designer coating'. Trans. Inst. Met. Finish. 2002, 80, B4-B7.
(8) Scorciapino, M. A. Concentration Depth-Profile Reconstruction from ARXPS Data Using the Maximum Entropy Method Characterization of Surface Films Formed on Nil8P Alloy. Ph.D. Thesis, University of Cagliari, Monserrato, CA, Italy, January 2008. http://veprints.unica.it/68/.

(9) Sharma, A.; Singh, A. K. Electroless Ni-P-PTFE- $\mathrm{Al}_{2} \mathrm{O}_{3}$ Dispersion Nanocomposite Coating for Corrosion and Wear Resistance. J. Mater. Eng. Perform. 2014, 23, 142-151.

(10) Crobu, M.; Scorciapino, M. A.; Elsener, B.; Rossi, A. The corrosion resistance of electroless deposited nano-crystalline Ni-P alloys. Electrochim. Acta 2008, 53, 3364-3370.

(11) Ratzker, M.; Lashmore, D. S.; Pratt, K. W. Electrodeposition and corrosion performance of nickel-phosphorus amorphous alloys. Plat. Surf. Finish. 1986, 73, 74-82.

(12) Królikowski, A.; Butkiewicz, P. Anodic behavior of Ni-P alloys studied by impedance spectroscopy. Electrochim. Acta 1993, 38, 19791983.

(13) Bielinski, J.; Krolikowski, A.; Kedzierska, I.; Stokarski, W. Compositional and structural factors in corrosion characteristics of $\mathrm{Ni}$ P deposits. ACH - Models Chem. 1995, 132, 685.

(14) Królikowski, A.; Karbownicka, B.; Jaklewicz, O. Anodic dissolution of amorphous Ni-P alloys. Electrochim. Acta 2006, 51, 6120-6127.

(15) Bozzini, A.; Lenardi, C.; Serra, M.; Fanigliulo, M. Elecrochemical and X-ray photoelectron spectroscopy investigation into anodic behaviour of electroless $\mathrm{Ni}-9-5 \mathrm{wt}-\% \mathrm{P}$ in acidic chloride environments. Br. Corros. J. 2002, 37, 173-181.

(16) Ashassi-Sorkhabi, H.; Rafizadeh, S. H. Effect of coating time and heat treatment on structures and corrosion characteristics of electroless Ni-P alloy deposits. Surf. Coat. Technol. 2004, 176, 318326.

(17) Burstein, G. T. Examination of passive films on iron-nickel alloys by Auger Electron spectroscopy. Corrosion 1981, 37, 549.

(18) Kawashima, A.; Asami, K.; Hashimoto, K. An XPS study of anodic behavior of amorphous nickel-phosphorus alloys containing chromium, molybdenum or tungsten in $1 \mathrm{M} \mathrm{HCl}$. Corros. Sci. 1984, 24, 807-823.

(19) Diegle, R. B.; Sorensen, N. R.; Nelson, G. C. Dissolution of glassy Ni-P alloys in $\mathrm{H}_{2} \mathrm{SO}_{4}$ and $\mathrm{HCl}$ electrolytes. J. Electrochem. Soc. 1986, 133, 1769-1776.

(20) Diegle, R. B.; Sorensen, N. R.; Clayton, C. R.; Helfand, M. A.; $\mathrm{Yu}, \mathrm{Y}$. C. An XPS investigation into the passivity of an amorphous Ni20 P alloy. J. Electrochem. Soc. 1988, 135, 1085-1092.

(21) Salvago, G.; Fumagalli, G. Electroless nickel-defects, passivity and localized corrosion. Met. Finish. 1987, 85, 31-35.

(22) Królikowski, A. Nature of anodic dissolution of amorphous Ni-P alloys. Mater. Sci. Forum 1995, 185-188, 799-808.

(23) Rossi, A.; Elsener, B. In Characterization of Surface Films on FeCrPC Alloys by XPS and X-ray Excited Auger Peaks; Marcus, P., Baroux, B., Keddam, M. Eds.; Surface Modifications of Passive films; EFC Publication No. 12 - The Institute of Materials: U.K., 1994; pp $6-11$

(24) Elsener, B.; Rossi, A. Interface between passive film and substrate-its role in corrosion resistance of alloys. Metall. Found. Eng. 1997, 23, 157-168.

(25) Elsener, B.; Crobu, M.; Scorciapino, M. A.; Rossi, A. Electroless deposited $\mathrm{Ni}-\mathrm{P}$ alloys: corrosion resistance mechanism. J. Appl. Electrochem. 2008, 38, 1053-1060.

(26) Opila, R. L.; Eng, J. J. R. Thin films and interfaces in microelectronics: composition and chemistry as function of depth. Prog. Surf. Sci. 2002, 69, 125-163.

(27) Hofmann, S. Depth Profiling in AES and XPS. In Practical Surface Analysis by Auger and X-Ray Photoelectron Spectroscopy, 2nd ed.; Briggs, D., Seah, M. P., Eds.; Wiley \& Sons Ltd.: West Sussex, 1990; pp 143-199.

(28) Rossi, A.; Elsener, B. XPS analysis of passive films on the amorphous alloy Fe70Cr10P13C7: effect of the applied potential. Surf. Interface Anal. 1992, 18, 499-504. 
(29) Tougaard, S. Surface nanostructure determination by x-ray photoemission spectroscopy peak shape analysis. J. Vac. Sci. Technol., A 1996, 14, 1415-1423. http://www.quases.com/

(30) Tougaard, S. Accuracy of the non-destructive surface nanostructure quantification technique based on analysis of the XPS and AES peak shape. Surf. Interface Anal. 1998, 26, 249-269.

(31) Cumpson, P. J. Angle-resolved XPS and AES: depth-resolution limits and a general comparison of properties of depth-profile reconstruction methods. J. Electron Spectrosc. Relat. Phenom. 1995, $73,25-52$.

(32) Holloway, P. H.; Bussing, T. D. Quantitative surface analysis of layered materials. Surf. Interface Anal. 1992, 18, 251-256.

(33) Smith, G. C.; Livesey, A. K. Maximum entropy: A new approach to non-destructive deconvolution of depth profiles from angledependent XPS. Surf. Interface Anal. 1992, 19, 175-180.

(34) Livesey, A. K.; Smith, G. C. The determination of depth profiles from angle-dependent XPS using maximum entropy data analysis. J. Electron Spectrosc. Relat. Phenom. 1994, 67, 439-461.

(35) Chang, J. P.; et al. Profiling nitrogen in ultrathin silicon oxynitrides with angle-resolved x-ray photoelectron spectroscopy. J. Appl. Phys. 2000, 87, 4449-4455.

(36) Champaneria, R.; Mack, P.; White, R.; Wolstenholme, J. Nondestructive analysis of ultrathin dielectric films. Surf. Interface Anal. 2003, 35, 1028-1033.

(37) Splinter, S. J.; McIntyre, N. S. Resolution enhancement of x-ray photoelectron spectra by maximum entropy deconvolution. Surf. Interface Anal. 1998, 26, 195-203.

(38) Olla, M.; Navarra, G.; Elsener, B.; Rossi, A. Nondestructive indepth composition profile of oxy-hydroxide nanolayers on iron surfaces from ARXPS measurement. Surf. Interface Anal. 2006, 38, 964-974.

(39) Yonamoto, Y. Application of Maximum Entropy Method to Semiconductor Engineering. Entropy 2013, 15, 1663-1689.

(40) Scorciapino, M. A.; Navarra, G.; Elsener, B.; Rossi, A. Nondestructive surface depth profiles from angle resolved $\mathrm{x}$-ray photoelectron spectroscopy data using the Maximum Entropy Method. I. A new protocol. J. Phys. Chem. C 2009, 113, 21328-21337.

(41) Crobu, M.; Rossi, A.; Mangolini, F.; Spencer, N. D. Chainlength-identification strategy in zinc polyphosphate glasses by means of XPS and ToF-SIMS. Anal. Bioanal. Chem. 2012, 403, 1415-1432.

(42) Heuberger, R.; Rossi, A.; Spencer, N. D. Pressure dependence of ZnDTP tribochemical film formation: a combinatorial approach. Tribol. Lett. 2007, 28, 209-222.

(43) Franke, R.; Chassé, T.; Streubel, P.; Meisel, A. Auger parameters and relaxation energies of phosphorus in solid compounds. J. Electron Spectrosc. Relat. Phenom. 1991, 56, 381-388.

(44) Wagner, C. D.; Joshi, A. The Auger parameter, its utility and advantages: a review. J. Electron Spectrosc. Relat. Phenom. 1988, 47, 283-313.

(45) Moretti, G. Auger parameter and Wagner plot in the characterization of chemical states by X-ray photoelectron spectroscopy: a review. J. Electron Spectrosc. Relat. Phenom. 1998, 95, 95-144.

(46) Van der Kouwe, E. T. EIS as a means of evaluating electroless nickel deposits. Electrochim. Acta 1993, 38, 2093-2097.

(47) Gunter, P. L. J.; Gijzeman, O. L. J.; Niemantsverdriet, J. W. Surface roughness effects in quantitative XPS: magic angle for determining overlayer thickness. Appl. Surf. Sci. 1997, 115, 342-346.

(48) Oswald, S.; Oswald, F. Modeling of surface roughness for ARXPS. Phys. Status Solidi C 2007, 4, 1817-1821.

(49) Spikes, H. A. The history and mechanisms of ZDDP. Tribol. Lett. 2004, 17, 469-489.

(50) Salvago, G.; Fumagalli, G.; Brunella, F. Corrosion behaviour of electroless Ni-P coatings in chloride-containing environments. Surf. Coat. Technol. 1989, 37, 449-460.

(51) Belin, E.; Traverse, A.; Szàsz, A.; Machizaud, F. Densities of states in amorphous Ni-P alloys. Influence of medium-range order. J. Phys. F: Met. Phys. 1987, 17, 1913-1923.
(52) Van Muylder, J.; Pourbaix, M. Phosphorus. In Atlas of Electrochemical Equilibria in Aqueous Solutions, 1st ed.; Pourbaix, M., Ed.; Pergamon Press Ltd: Oxford, 1966; pp 506-515.

(53) Seah, M. P. Summary of ISO/TC 201 Standard: VII ISO 15472: 2001-surface chemical analysis-x-ray photoelectron spectrometerscalibration of energy scales. Surf. Interface Anal. 2001, 31, 721-723.

(54) Cumpson, P. J. In Angle-Resolved X-ray Photoelectron Spectroscopy; Briggs, D., Grant, J. T., Eds.; Surface Analysis by Auger and X-ray Photoelectron Spectroscopy; Surface Spectra and IM Publications: U.K., 2003; p 651.

(55) Cumpson, P. J.; Seah, M. P. Elastic scattering corrections in AES and XPS. II. Estimating attenuation lengths and conditions required for their valid use in overlayer/substrate experiments. Surf. Interface Anal. 1997, 25, 430-446.

(56) Watts, J. F.; Wolstenholme, J. Compositional Depth Profiling. In An Introduction to Surface Analysis by XPS and AES, 1st ed.; Watts, J. F., Wolstenholme, J., Eds.; John Wiley and Sons Ltd: Chichester, 2003; pp 89-91.

(57) Scofield, J. H. Hartree-Slater subshell photoionization crosssections at 1254 and $1487 \mathrm{eV}$. J. Electron Spectrosc. Relat. Phenom. 1976, 8, 129-137.

(58) Reilman, R. F.; Msezane, A.; Manson, S. T. Relative intensities in photoelectron spectroscopy of atoms and molecules. J. Electron Spectrosc. Relat. Phenom. 1976, 8, 389-394.

(59) Gries, W. H. A universal predictive equation for the Inelastic Mean Free Pathlengths of X-ray Photoelectrons and Auger Electrons. Surf. Interface Anal. 1996, 24, 38-50.

(60) Powell, C. J.; Jablonski, A. NIST Electron Inelastic-Mean-FreePath Database, version 1.1; NIST, 2000. http://www.nist.gov/srd/ nist71.htm.

(61) Jablonski, A.; Powell, C. J. The electron attenuation length revisited. Surf. Sci. Rep. 2002, 47, 33-91.

(62) Jablonski, A. Quantitative AES: Via the inelastic mean free path or the attenuation length? Surf. Interface Anal. 1990, 15, 559-566. 